\title{
P17 Comparisons of Carotid-femoral Pulse Wave Velocity Obtained from the Surface-distance Measurement and from the Population-derived Distance Formula: Associations with Macro- and Microvascular Alterations in Older Adults
}

\author{
Kunihiko Aizawa*, Francesco Casanova, Dave Mawson, Kim Gooding, Salim Elyas, Damilola Adingupu, \\ David Strain, Phillip Gates, Angela Shore
}

University of Exeter College of Medicine and Health, NIHR Exeter Clinical Research Facility, Exeter, UK

\begin{abstract}
Introduction: Inaccurate determination of arterial path-length by surface-distance (SD) measurement is an inherent source of error in calculating carotid-femoral pulse wave velocity (CFPWV). A recent development in calculating arterial path-length by a simple distance formula (DF) for CFPWV has been shown to not only remove intercentre measurement variability but also strengthen the association between CFPWV and traditional cardiovascular risk factors [1]. We determined whether the association of macro- and microvascular alterations with CFPWV derived from the distance formula (CFPWV-DF) would be stronger than that with CFPWV derived from the surface-distance measurement (CFPWV-SD).

Methods: CFPWV-DF and CFPWV-SD were obtained from 489 older adults (67.2 \pm 8.8 yrs, 154 F, 244 CVD). Macrovascular [carotid lumen diameter (LD), carotid inter-adventitial diameter (IAD), carotid intima-media thickness (IMT), carotid total plaque area, and ankle-brachial pressure index] and microvascular [reactive hyperaemia index and urinary albumin-creatinine ratio (UACR)] parameters were also obtained.

Results: CFPWV-DF was significantly lower than CFPWV-SD ( $9.27 \pm 2.38$ vs $9.88 \pm 2.55 \mathrm{~m} / \mathrm{s}, p<0.001)$, which resulted from a longer arterial path-length estimated by SD than DF ( $495.4 \pm 44.8$ vs $465.3 \pm 20.6 \mathrm{~mm}, p<0.001)$. The significant associations observed between CFPWV-SD and LD $(r=0.264)$, IAD $(r=0.303)$, and IMT $(r=0.165)$ were similarly observed between CFPWV-DF and LD $(r=0.253)$, IAD $(r=0.303)$, and IMT $(r=0.183$, all $p<0.001)$. This was also the case with UACR $(r=0.163$ and $r=0.141$, both $p=0.001$ ). Other parameters did not show any association in either CFPWV.
\end{abstract}

Conclusion: The association of macro- and microvascular alterations with CFPWV-DF was not stronger than that with CFPWV-SD, suggesting that arterial path-length determination methods may not influence interactions between vascular biomarkers and CFPWV.

\section{REFERENCE}

[1] Weir-McCall JR, Brown L, Summersgill J, Talarczyk P, Bonnici-Mallia M, Chin SC, et al. Development and validation of a path length calculation for carotid-femoral pulse wave velocity measurement: A TASCFORCE, SUMMIT, and caerphilly collaborative venture. Hypertension 2018;71:937-45.

(c) 2019 Association for Research into Arterial Structure and Physiology. Publishing services by Atlantis Press International B.V. This is an open access article distributed under the CC BY-NC 4.0 license (http://creativecommons.org/licenses/by-nc/4.0/). 